\title{
Estrutura e composição florística em área de Floresta Ombrófila Densa sob manejo florestal
}

\author{
Mathaus Messias Coimbra Limeira ${ }^{1 *}$ Yasmin Andrade Ramos ${ }^{1}$ Max Vinicios Reis de Sousa ${ }^{1}$ Maria \\ Cristina Bueno Coelho ${ }^{1}$ Maurilio Antonio Varavallo ${ }^{1}$ Yandro Santa Brigida Ataide ${ }^{1}$ André Ferreira \\ dos Santos ${ }^{1}$ Mauro Luiz Erpen ${ }^{1}$
}

${ }^{1}$ Universidade Federal do Tocantins, Rua Barcelona Sevilha, CEP 77402-970, Gurupi, TO Brasil R., Gurupi - TO, 77402-970

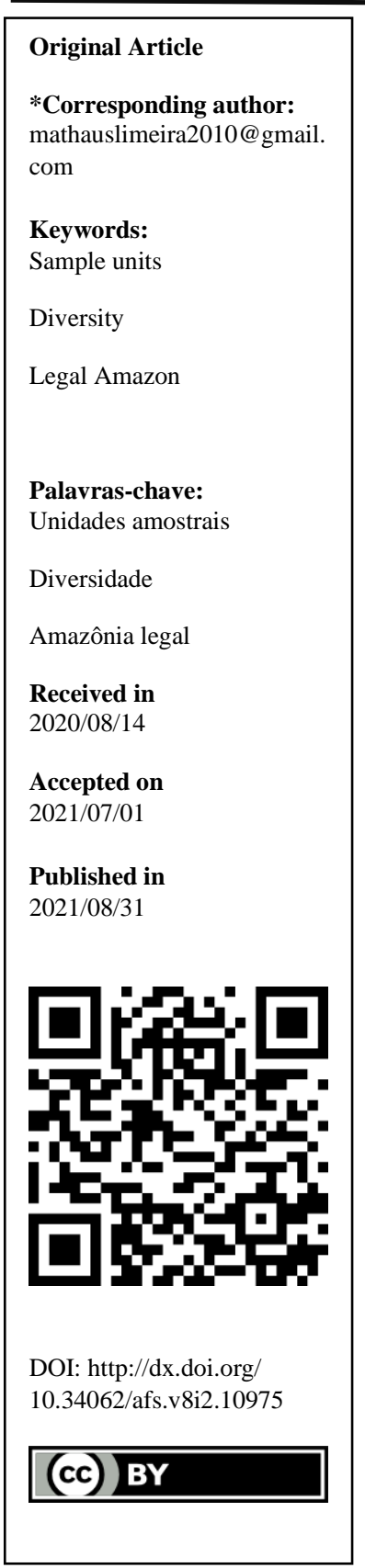

RESUMO: O propósito do presente trabalho é determinar a fitossociologia e florística de um fragmento de Floresta Ombrófila Densa sob manejo florestal na região de Portel, Pará. Foram amostrados 4 conglomerados, divididos em parcelas secundárias e terciárias totalizando 80 unidades amostrais de 0,4 hectares cada. Nestas foram medidos todos os indivíduos arbóreos com diâmetro a altura do peito (DAP), a 1,30 m do nível do solo, acima de $10 \mathrm{~cm}$ além de determinar a fitossociologia e realizar os cálculos dos parâmetros de equabilidade e similaridade florística. Foram registrados 1621 indivíduos, distribuídos em 35 famílias e 146 espécies. As famílias Fabaceae, Lecythidaceae e Sapotaceae foram as que obtiveram os maiores índices do valor de importância familiar e a maior representatividade de indivíduos. As espécies Eschweilera coriacea (DC.) S. A. Mori, Licania macrophylla Benth e Vouacapoua americana Aubl. foram as que tiveram os maiores IVI. A média do índice de variedade de Shannon entre as parcelas foi de 3,82 e o da equabilidade de Pielou 0,84 constatando que a região apresenta alta diversidade florística além de apresentar grande potencialidade para as atividades do manejo florestal.

\section{Floristic Structure and Composition in An Area of Dense Rainforest Under Forest Management}

ABSTRACT: The purpose of this work is to deliver the phytosociology and floristicity of a fragment of Dense Ombrophilous Forest under forest management in the region of Portel, Pará. Four clusters were sampled, divided into secondary and terciarias plots totaling 80 sampling units of 0,4 hectares each. In which all tree individuals with diameter at breast height (DAP), at $1,30 \mathrm{~m}$ from ground level, above $10 \mathrm{~cm}$ were measured, in addition to performing phytothegic recognition and elaborating the calculations of the paramemers of equability and floristic similarity. A total of 1621 individuals were recorded, distributed in 35 families and 146 species. The families Fabaceae, Lecythidaceae and Sapotaceae were the ones with the highest IVIF and the highest representativeness of individuals. The species Eschweilera coriacea (DC.) S.A. Mori, Licania macrophylla Benth., Vouacapoua americana Aubl were the ones with the highest IVI. The mean Shannon variety index among the plots was 3,82 and the Pielou Equability index was 0,84 , noting that the region has a high floristic variety and has great potential for forest management activities. 


\section{Introdução}

O Brasil é considerado um dos países de maior essência em diversidade mundial e apresenta um terço das florestas tropicais do mundo, ocupando aproximadamente 4,2 milhões de $\mathrm{km}$ (Silva et al. 2008). Estes ambientes florestais são formados pela flora rica e variada, sendo na maioria das vezes exclusivas de cada local (Oliveira e Amaral 2004). A Amazônia ocupa cerca de 50\% do território nacional (IBGE, 2012), apresenta a maior biodiversidade do planeta, incluindo a diversidade genética e de ecossistemas, oriunda tanto da ação antrópica, quanto da interação dos fatores ambientais das dadas fitofisionomias (florestas de várzea, terra firme, cerrado e restingas, entre outras), na qual se agrega uma enorme gama de espécies ainda desconhecidas pela ciência (Pereira et al. 2011). As florestas aluviais são aquelas que ficam periodicamente inundadas. Popularmente são conhecidas como várzeas (inundadas por rios de águas barrentas) e igapós (inundadas por rios de águas escuras) (Scudeller e Souza 2009). Além disso dominam nestes ambientes os latossolos distróficos e é possível observar também a figura de famílias de dissipação pantropical, existente por todo o continente como Fabaceae, Sapotaceae e Lecythidaceae, sendo a última considerada típica, ou seja, apresentam fisionomias marcantes nos locais em que são apresentadas (IBGE, 2012).

A partir de várias pesquisas realizadas na Amazônia, como os realizados por Almeida et al. (2012), Castro et al. (2018), Condé e Tonini (2013) e Lima Filho et al. (2001), pode-se reconhecer que os ambientes florestais de terra forte apresentam uma alta variedade, com uma grande ocorrência de espécies raras (poucas pessoas do mesmo tipo) e com uma similaridade com princípios bastante variáveis. As informações obtidas nessas análises são ferramentas fundamentais para a identificação da potencialidade do setor e das técnicas para o controle florestal apropriado (Castro et al. 2018).

$\mathrm{O}$ desenvolvimento de estudos que visam nortear tomadas de decisões por meio da análise da
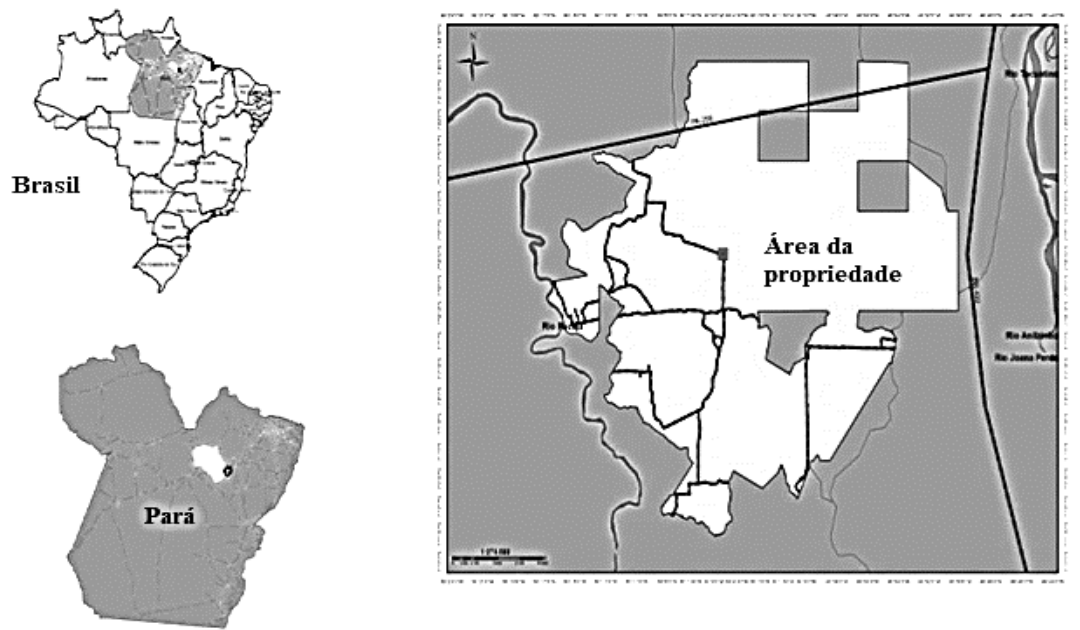

Figura 1. Localização da Fazenda Uberlândia no Estado do Pará, Brasil. estrutura da comunidade florestal é sem dúvida imprescindível para uso dos recursos naturais de forma sustentável. Para conservar a vegetação natural é preciso entender seu comportamento frente aos distúrbios naturais e antropogênicos (Batista et al. 2015). Levantamentos florísticos e fitossociológicos são ferramentas fundamentais para as pesquisas dos estágios futuros da vegetação, pois a hereditariedade de espécies florestais é formada através da conservação de mudanças florísticas e estruturais que ocorrem no ecossistema bem como a influência de ações antrópicas.

A hipótese aqui testada é de que o manejo florestal não altera o funcionamento natural da floresta nativa, embora possa ocorrer uma redução entre a relação espécie-individuo, as famílias nativas continuam presentes e a diversidade florística se mantém.

Nesse contexto, o objetivo desta pesquisa foi determinar a estrutura e a composição florística em uma área de Floresta Ombrófila Densa Aluvial, sob manejo florestal na Fazenda Uberlândia, no município de Portel, Pará.

\section{Material e Métodos}

Caracterização da área de estudo

A área de estudo está localizada na Fazenda Uberlândia, localizada na região do Alto Pacajá, a uma latitude 2059'37.78'’S e longitude $50^{\circ} 15^{\prime} 13.90^{\prime \prime} \mathrm{O}$. Compreende uma área de Floresta Ombrófila Densa Aluvial da Fazenda Uberlândia, situada na região do Alto Pacajá e abrange 4 municípios: Portel, Bagre, Baião e Oeiras do Pará. A área total da fazenda é de 153.115,02 hectares, 120.017 hectares correspondem a área de cobertura florestal. A mesma é de propriedade do Grupo Martins Agropecuária S/A sendo arrendada para a empresa LN Guerra Industria e Comércio de Madeiras LTDA que tem como objetivo básico o uso dos recursos florestais através das práticas de manejo florestal de impacto reduzido desde meados de 2012. 
$\mathrm{Na}$ área o clima é classificado por Köppen como "Am”, tropical úmido (Alvares et al. 2013), caracterizado por proporcionar chuvas do tipo monção e uma estação seca de baixa permanência, mas com umidade suficiente para suprir a floresta tropical. Recebe anualmente $2.400 \mathrm{~mm}$ de chuva, a temperatura média do ar é de $26^{\circ} \mathrm{C}$ e a umidade relativa superior a $85 \%$ (Fisch et al. 1998).

O solo predominante da região é o Latossolo Amarelo Distrófico, textura argilosa e o Latossolo Amarelo Distrófico Concrecionário, textura argilosa. A área da propriedade está localizada na região classificada como planalto rebaixado da Amazônia ou Baixo Amazonas, numa região de baixos platôs com vegetação emergente (IBGE, 2012). O solo é inclinado, com uma parte baixa (barranco) mais exposta à correnteza do rio e sujeita à erosão e sedimentação (Perigolo 2014). A área da propriedade está localizada na região classificada como planalto rebaixado da Amazônia ou Baixo Amazonas, numa região de baixos platôs com vegetação de emergente (Dos Santos et al. 2013).

$\mathrm{Na}$ área da propriedade foram encontrados 5 tipos de formações florestais que foram classificadas e descritas com base na terminologia adotada conforme IBGE (2012). A área onde o estudo foi conduzido foi a de Floresta Ombrófila Densa Aluvial, com uma área de 29.716,33 ha. Dentre todas as florestas inundáveis do mundo, a Floresta Ombrófila Densa Aluvial Amazônica possui a maior quantidade em espécies do reino Plantae (Junk et al. 2011; Wittmann et al. 2013).

De qualquer forma, a Floresta Ombrófila Densa Aluvial contribui muito para a variedade florística total, uma vez que a constituição de espécies se modifica entre as regiões e localmente, em ofício de pequenas variações topográficas bem como do gradiente de inundação (Wittmann et al. 2013). É constituída pelas plantas com dossel de altura média entre 15 a $20 \mathrm{~m}$, e emergentes de 25 a
$30 \mathrm{~m}$. Constantemente borda e dossel são cobertos por trepadeiras, com desigualdade de uma estrutura considerada fechada aberta, no qual se concentram espécies de Cecropia. A altura dos rios amazônicos pode oscilar em média de 7 a $13 \mathrm{~m}$ ao longo do ano e, dependendo da profundidade e espaço de tempo da inundação, as variações topográficas provocam mudanças dramáticas nos seres vegetais, com preeminência nas comunidades arbustivas mais baixas do terreno, e outra comunidade arbórea nos graus mais altos (Wittmann et al. 2013).

\section{Teoria da amostragem}

Segundo recomendação De Queiroz et al. (2011), as unidades amostradas seguiram o desenho esquemático em forma de Cruz de Malta (conforme mostra a figura 2), onde cada conglomerado (parcela) teve forma retangular de $5 \mathrm{~km}$ x $5 \mathrm{~km}$, onde é composta por 5 unidades secundárias, também organizadas em cruz a partir do centro, de tamanho $500 \mathrm{~m}$ x 500m e equidistantes $200 \mathrm{~m}$ entre si, totalizando 8 ha. Estas unidades secundárias foram compostas por 4 unidades terciárias com dimensão de área retangular de 0,4 ha $(20 \mathrm{~m}$ x $200 \mathrm{~m})$, distantes $50 \mathrm{~m}$ do ponto central, formando um total de 80 unidades amostrais.

A localização das unidades secundárias e terciárias foi realizada através do uso de uma bússola, onde se mediu os ângulos que orientaram a delimitação dessas unidades na área. A referência para esta delimitação foi o ponto central das unidades primárias.

A área amostrada seguiu o critério da porcentagem da área da população a ser amostrada, conforme descrito por Soares et al. (2006), ou seja, na área em questão obtendo-se assim um $\mathrm{N}$ inicial de 32 hectares ( 80 unidades amostrais $\times 0,4$ hectares $=$ 32 hectares) o que isso representa a cerca de $0,10 \%$ da área total.

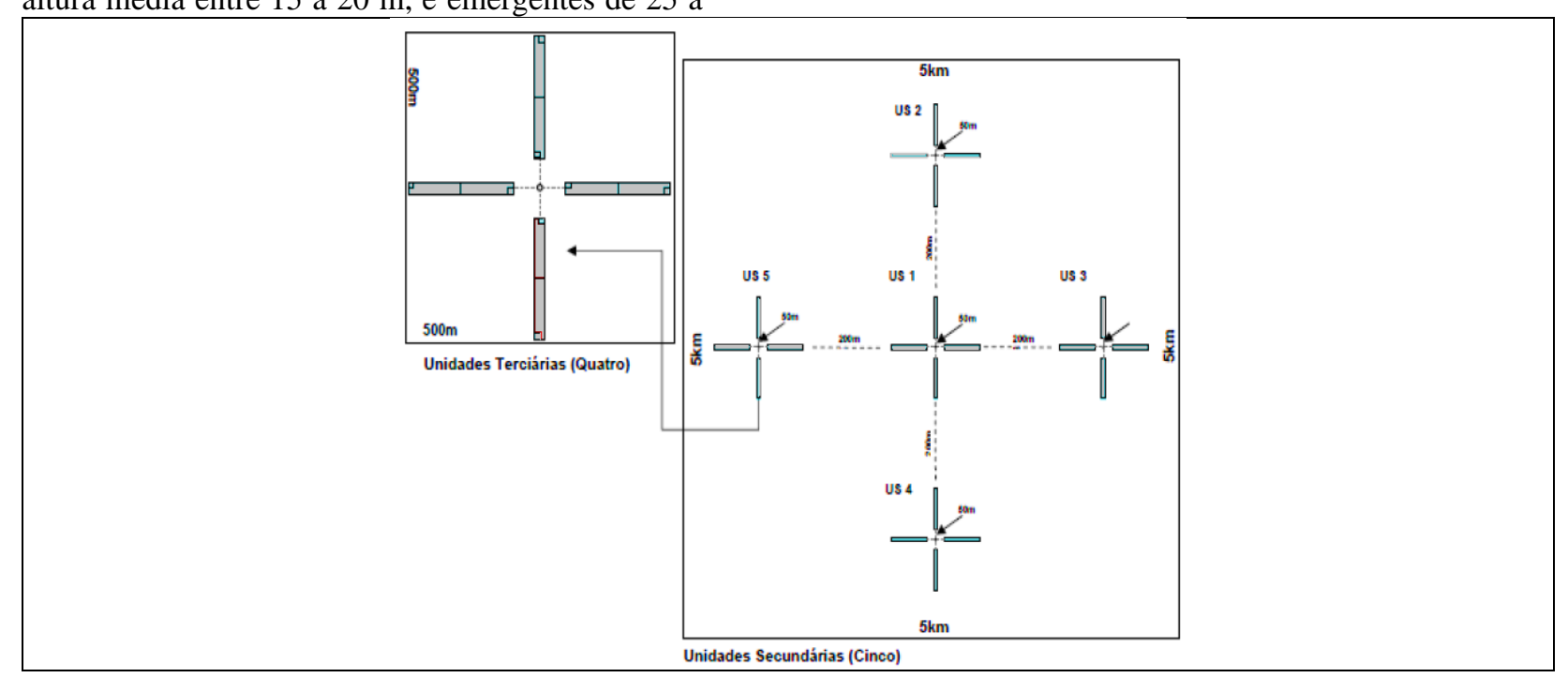

Figura 2. Desenho esquemático dos conglomerados (parcelas amostrais) distribuídas na área de estudo. 


\section{Limeira et al.}

Nas unidades primárias foi feito um inventário onde foram medidos todos os indivíduos arbóreos com DAP (diâmetro à altura do peito em $\mathrm{cm}$ ) superior a $10 \mathrm{~cm}$. Além do DAP, a altura comercial foi estimada de forma visual para cada indivíduo avaliado.

Ao longo da coleta das espécies, as espécies foram identificadas somente pelo conhecimento popular. Sucessivamente a constatação do nome científico, dos materiais coletados, foi executado por meio da semelhança com exsicatas do Herbário do Museu Paraense Emilio Goeldi (MPEG), estado do Pará. O sistema de classificação adotado foi o The Angiosperm Phylogeny Group (APG III, 2009). Para a análise estrutural da floresta determinou-se os parâmetros conforme descrito por Müller-Dombois e Ellemberg (1974) de acordo com a tabela 1.

Tabela 1. Parâmetros fitossociológicos e índices para determinar a estrutura e composição florística.

\begin{tabular}{|c|c|c|}
\hline & Formula: & Em que: \\
\hline Frequência Absoluta & $F A=\frac{u i}{u t} \times 100$ & $\begin{array}{l}\text { ui = número de unidades amostrais em que a i-ésima } \\
\text { espécie ocorre; ut }=\text { número total de unidades } \\
\text { amostrais. }\end{array}$ \\
\hline Frequência Relativa & $F R=\frac{F A}{\sum F A} \times 100$ & $\begin{array}{l}\text { FA = frequência absoluta da i-ésima espécie na } \\
\text { comunidade vegetal; FR = frequência relativa da i- } \\
\text { ésima espécie na comunidade vegetal. }\end{array}$ \\
\hline Densidade Absoluta & $D A=\frac{n}{A}$ & $\begin{array}{l}\mathrm{n}=\text { número de indivíduos da i-ésima espécie na } \\
\text { amostragem; } \mathrm{A}=\text { área total amostrada em hectare. }\end{array}$ \\
\hline Densidade Relativa & $D R=\frac{D A}{D T} \times 100$ & $\begin{array}{l}\mathrm{DA}=\text { densidade absoluta da i-ésima espécie, em } \\
\text { número de indivíduos por hectare; DR = densidade } \\
\text { relativa }(\%) \text { da i-ésima espécie. }\end{array}$ \\
\hline Dominância Absoluta & $D o A=\frac{A B}{A}$ & $\begin{array}{l}\mathrm{AB}=\text { área basal da i-ésima espécie, em } \mathrm{m}^{2} \text {, na área } \\
\text { amostrada; } \mathrm{A}=\text { área amostrada, em hectare; }\end{array}$ \\
\hline Dominância Relativa & $D o R=\frac{D o A}{D o T} \times 100$ & $\begin{array}{l}\text { DoA = dominância absoluta da i-ésima espécie, em } \\
\mathrm{m}^{2} / \text { ha; } \mathrm{DoR}=\text { dominância relativa }(\%) \text { da i-ésima } \\
\text { espécie. }\end{array}$ \\
\hline $\begin{array}{l}\text { Índice de Valor de } \\
\text { Importância }\end{array}$ & $I V I=(D R+F R i+D o R)$ & $\begin{array}{l}\text { DoR = dominância relativa }(\%) \text { da i-ésima espécie. } \\
\mathrm{DR}=\text { densidade relativa }(\%) \text { da i-ésima espécie. FR } \\
=\text { frequência relativa da i-ésima espécie na } \\
\text { comunidade vegetal. }\end{array}$ \\
\hline $\begin{array}{l}\text { Índice de Shannon- } \\
\text { Weaver }\end{array}$ & $H^{\prime}=\sum^{S}[p i \ln (p i)]$ & $\mathrm{pi}=\mathrm{ni} / \mathrm{N}$ \\
\hline $\begin{array}{l}\text { Equabilidade de } \\
\text { Pielou }\end{array}$ & $J=\frac{H^{\prime}}{\ln (S)}$ & lon-Weaver \\
\hline Índice De Sorensen & $S O i j=\frac{2 c}{a+b}$ & $\begin{array}{l}\text { c é o número de espécies comuns as duas parcelas; a } \\
\text { é número de espécies ocorrentes na parcela } 1 ; \text { e b é } \\
\text { o número de espécies ocorrentes na parcela } 2 \text {. }\end{array}$ \\
\hline Índice de Morisita & $I M=q \frac{\sum_{i=1}^{q} X i(X i-1)}{T(T-1)}$ & $\begin{array}{l}\mathrm{q}=\text { número de parcelas; } \mathrm{x}=\text { número de indivíduos } \\
\mathrm{i} \text {-ésima parcela; } \mathrm{T}=\text { número total de indivíduos } \\
\text { amostrados. }\end{array}$ \\
\hline Número de classes & $N=1+3,3 \log (n)$ & $\begin{array}{l}\mathrm{N}=\text { número de classes, } \mathrm{n} \text { é o número de indivíduos } \\
\text { amostrais. }\end{array}$ \\
\hline Qui-quadrado & $X^{2}=\frac{n * \sum x^{2}}{N}-N$ & $\begin{array}{l}X^{2}=\text { valor do qui quadrado, } \mathrm{n} \\
=\text { número total de parcelas amostradas; } \mathrm{N} \\
=\text { número total de indivíduos; } x^{2} \\
=\text { quadrado do número de indivíduos por parcela }\end{array}$ \\
\hline $\begin{array}{l}\text { Coeficiente de } \\
\text { Liocourt }\end{array}$ & $q=\frac{e^{(\beta 0+\beta 1 X i)}}{e^{(\beta 0+\beta 1 X i+1)}}$ & $\begin{array}{l}q=\text { coeficiente de Liocurt, } \mathrm{Xi}=\text { Frequências de uma } \\
\text { classe de diâmetro qualquer, } \mathrm{Xi}+1=\text { Frequência } \\
\text { imediata acima }\end{array}$ \\
\hline
\end{tabular}

Os valores adquiridos por este foram interpretados, por meio de agrupamentos por média de grupo (UPGMA), representados em dendrograma que foi obtido por meio do Software MVSP.

\section{Estrutura vertical}

Com a análise da posição fitossociológica obtemos a importância da espécie e a sua participação nos estratos verticais da floresta. Neste estudo foram utilizados três estratos de altura para estimar a posição sociológica absoluta por espécie na área florestal, conforme descrito por Souza (2003), 
em que: $\mathrm{Hm}=$ média das alturas totais $(\mathrm{Ht})$ dos indivíduos amostrados; $\mathrm{S}=$ desvio padrão das alturas totais dos indivíduos amostrados; HT = altura total da $\mathrm{j}$ - ésima árvore. Estrato inferior (E1): Árvore com HT < $(\mathrm{Hm}-1 \mathrm{~S})$; Estrato médio (E2): Árvore com $(\mathrm{Hm}-1 \mathrm{~S}) \leq \mathrm{HT}<(\mathrm{Hm}+1 \mathrm{~S})$; Estrato superior (E3): Árvore com $\mathrm{HT} \geq(\mathrm{Hm}-1 \mathrm{~S})$.

Feita a classificação das árvores em seus respectivos estratos (inferior, médio ou superior), as posições sociológicas absoluta e relativa (PSA e PSR, respectivamente) foram obtidas pelas seguintes expressões:

$$
\begin{aligned}
& P S A=\sum_{J-1}^{J}\left(\frac{N j}{N}\right) X N i j \\
& P S R=\frac{P S A}{\sum P S A} \times 100
\end{aligned}
$$

Onde: PSA = posição sociológica absoluta d $\mathrm{i}$ - ésima espécie; $\mathrm{Nj}$ = número de indivíduos do $\mathrm{i}-$ ésimo estrato; $\mathrm{N}=$ número total das espécies, em todos os estratos; $\mathrm{Nij}=$ número de indivíduos da $\mathrm{i}-$ ésima espécie no $\mathrm{j}$ - ésimo estrato de altura; PSR = posição sociológica relativa da i-ésima espécie, em percentagem.

Os cálculos de todos os parâmetros avaliados nas estruturas vertical e horizontal foram feitos por meio de planilhas eletrônicas do Software Microsoft Excel 2011.

\section{Resultados e Discussão}

Na tabela 2 estão apresentados os valores da estatística descritiva para as variáveis Diâmetro à altura do peito $(\mathrm{cm})$, altura comercial $(\mathrm{m})$ e área basal $\left(\mathrm{m}^{2}\right)$ áreas estudadas. A dispersão dos dados para todas as variáveis é considerada alta $(\mathrm{CV} \%$ > 20), indicando alta variação característica de áreas nativas (Gomes 1990). Com os valores obtidos dos coeficientes de assimetria para todas as variáveis estudadas apresentaram uma distribuição assimétrica positiva, ou seja, onde a maior frequência dos diâmetros mensurados está do lado esquerdo da distribuição. Já para o coeficiente de curtose, podemos definir que a maioria das espécies que compõem a área florestal apresentaram uma distribuição leptocúrtica, ou seja, da função de distribuição mais afunilada com um pico mais alto do que a distribuição normal possui uma curva mais achatada no topo em relação à curva normal. Como $71,19 \%$ dos indivíduos amostrados estão entre a média e \pm desvio padrão, indica que o fragmento florestal analisado é considerado como uma distribuição normal.

Tabela 2. Estatísticas descritivas para as variáveis DAP (diâmetro a altura do peito em centímetros), Hc (altura comercial em metros) e AB (área basal em metros quadrados) para área estudada no município de Portel - PA.

\begin{tabular}{cccc}
\hline Variável & DAP $(\mathbf{c m})$ & $\mathbf{H c}(\mathbf{m})$ & $\mathbf{A B} \mathbf{( m}^{\mathbf{2}} \mathbf{)}$ \\
\hline Média & 45,84 & 11,31 & 0,1996 \\
Erro padrão & 0,52 & 0,09 & 0,0049 \\
Coeficiente de Variação & 45,76 & 31,82 & 98,37 \\
Desvio padrão & 20,98 & 3,60 & 0,1963 \\
Variância da amostra & 439,97 & 12,96 & 0,0385 \\
Curtose & 1,92 & 1,34 & 12,5277 \\
Assimetria & 1,06 & 0,94 & 2,8383 \\
Intervalo & 145,79 & 20,00 & 1,9025 \\
Mínimo & 10,19 & 5,00 & 0,0081 \\
Máximo & 155,97 & 25,00 & 1,9107 \\
Nível de confiança $(95.0 \%)$ & 1,02 & 0,18 & 0,0096 \\
\hline
\end{tabular}

Composição florística

Nas 4 unidades amostrais distribuídas aleatoriamente foram encontrados 1621 indivíduos, distribuídos em 34 famílias, 98 gêneros e 166 espécies. As famílias com maior riqueza de espécies foram Fabaceae (41), Sapotaceae (11) e Lecythidaceae (8). As 20 espécies com maior Índice de Valor de Importância (IVI) totalizaram cerca de $58,6 \%$ de todos os indivíduos amostrados (tabela 3 ).

Tabela 3. Distribuição em ordem decrescente das 20 espécies que mais se destacaram em Índice de Valor de

\begin{tabular}{|c|c|c|c|c|c|c|c|c|c|}
\hline Espécies & $\mathbf{N}$ & FA & FR & DA & DR & DoA & DoR & IVI & IM \\
\hline Eschweilera coriacea (DC.) S. A. Mori. & 198 & 0,80 & 6,00 & 6,27 & 12,21 & 0,99 & 9,65 & 27,86 & RG \\
\hline Licania macrophylla Benth. & 128 & 0,63 & 4,76 & 4,05 & 7,90 & 0,57 & 5,52 & 18,18 & RG \\
\hline Vouacapoua americana Aubl. & 72 & 0,52 & 3,90 & 2,28 & 4,44 & 0,31 & 3,07 & 11,42 & $\mathrm{AG}$ \\
\hline Sagotia brachysepala (Müll.Arg.) Secco. & 66 & 0,38 & 2,86 & 2,09 & 4,07 & 0,22 & 2,17 & 9,10 & RG \\
\hline Inga heterophylla Willd. & 55 & 0,41 & 3,05 & 1,74 & 3,39 & 0,21 & 2,00 & 8,44 & RG \\
\hline Alexa grandiflora Ducke. & 49 & 0,41 & 3,05 & 1,55 & 3,02 & 0,55 & 5,37 & 11,44 & $\mathrm{AG}$ \\
\hline Macrosamanea pubiramea (Steud.) Barneby \& J.W. Grimes. & 49 & 0,38 & 2,86 & 1,55 & 3,02 & 0,30 & 2,92 & 8,80 & RG \\
\hline Neoxythece elegans (A.DC.) Aubrév. & 36 & 0,18 & 1,33 & 1,14 & 2,22 & 0,14 & 1,33 & 4,88 & RG \\
\hline
\end{tabular}
Importância (IVI) na área de floresta estudada. 


\section{Limeira et al.}

\begin{tabular}{llllllllll}
\hline Eschweilera odora (Poepp.) Miers. & 34 & 0,19 & 1,43 & 1,08 & 2,10 & 0,17 & 1,63 & 5,16 & $\mathrm{AG}$ \\
\hline Eschweilera amara Mart. & 33 & 0,24 & 1,81 & 1,04 & 2,04 & 0,15 & 1,45 & 5,30 & $\mathrm{AG}$ \\
\hline Goupia glabra Aubl. & 31 & 0,32 & 2,38 & 0,98 & 1,91 & 0,37 & 3,57 & 7,86 & $\mathrm{RG}$ \\
\hline Anacardium giganteum W. Hancock ex Engl. & 29 & 0,32 & 2,38 & 0,92 & 1,79 & 0,29 & 2,84 & 7,01 & $\mathrm{RG}$ \\
\hline Macrolobium limbatum Spruce ex Benth. & 28 & 0,27 & 2,00 & 0,89 & 1,73 & 0,21 & 2,00 & 5,73 & $\mathrm{RG}$ \\
\hline Eschweilera alata A.C.Sm. & 26 & 0,20 & 1,52 & 0,82 & 1,60 & 0,15 & 1,51 & 4,64 & $\mathrm{AG}$ \\
\hline Manilkara dardanoi Ducke. & 23 & 0,23 & 1,71 & 0,73 & 1,42 & 0,18 & 1,73 & 4,86 & $\mathrm{RG}$ \\
\hline Syzygiopsis oppositifolia Ducke. & 21 & 0,22 & 1,62 & 0,66 & 1,30 & 0,22 & 2,13 & 5,04 & $\mathrm{AG}$ \\
\hline Piptadenia gonoacantha (Mart.) J. F. Macbr. & 19 & 0,18 & 1,33 & 0,60 & 1,17 & 0,17 & 1,61 & 4,12 & $\mathrm{AG}$ \\
\hline Dinizia excelsa Ducke. & 18 & 0,15 & 1,14 & 0,57 & 1,11 & 0,37 & 3,57 & 5,82 & $\mathrm{RG}$ \\
\hline Iryanthera grandis Ducke. & 18 & 0,18 & 1,33 & 0,57 & 1,11 & 0,23 & 2,30 & 4,74 & $\mathrm{RG}$ \\
\hline Couratari oblongifolia Ducke et R. Knuth. & 17 & 0,19 & 1,43 & 0,54 & 1,05 & 0,19 & 1,88 & 4,36 & $\mathrm{AG}$ \\
\hline
\end{tabular}

Em que: $(\mathrm{N})$ = número indivíduos por espécie, $(\mathrm{FA})=$ frequência absoluta, $(\mathrm{FR})=$ frequência relativa, $(\mathrm{DA})=$ densidade absoluta, $(D R)=$ densidade relativa, $($ DoA $)=$ dominância absoluta, $($ DoR $)=$ dominância relativa, $($ IM) $=$ Índice de Morisita, $(\mathrm{AG})=$ Agregado, $(\mathrm{RG})=$ Regular

Oliveira e Amaral (2004) destacaram que o Índice de Valor de Importância (IVI) pode ser utilizado como uma importante ferramenta em planos de manejo como um indicador da importância ecológica das espécies, através da influência das espécies de maior frequência e dominância em processos de equilíbrio da flora e auxílio na conservação da fauna, disponibilizando abrigo e alimentação.

Quanto ao padrão de distribuição espacial das espécies através do Índice de Morisita (IM), considerando toda a população é de 1,01 considerado agregado, o que reflete quando calculamos o índice por espécie onde 48,29\% do total das espécies obteve um padrão agregado, sendo ocasionado principalmente pela remoção de árvores comerciais na área e 34,013\% é considerado regular. Para Lamprecht (1962) uma distribuição regular (maior número de indivíduos nas classes inferiores) é a maior garantia para a existência e sobrevivência das espécies. Este índice agregado é confirmado pelo teste do $X^{2}$ em que o valor qui-quadrado foi maior
$(22,58)$ que o tabelado $(7,81)$ a área tenderá a um padrão de distribuição agregada.

Classificação ecológica e estrutura das espécies

As 10 espécies de maiores IVI foram Eschweilera coriacea, Licania macrophylla, Alexa grandiflora, Vouacapoua americana, Sagotia brachysepala, Macrosamanea pubiramea, Inga heterophylla, Goupia glabra, Anacardium giganteum e Dinizia excelsa, juntas estas espécies representaram $38,6 \%$ do IVI total de indivíduos amostrados (Figura 3).

Dente as 146 espécies inventariadas nesta tipologia, 77 espécies obtiveram um IVI inferior a 1, dentre elas Buchenavia capitata (Vahl) Eichler, Hevea brasiliensis L, e Lecythis alutacea (A.C.Sm.) S.A. Mori. A espécie Eschweilera coriacea apresentou nas duas áreas maior domínio de habitat, considerada assim como a de maior importância. As espécies de maiores IVI, em sua maioria apresentaram um padrão regular.



Figura 3. Parâmetros fitossociológicos das 10 espécies amostradas com maior índice de valor de importância. DR, DoR, e FR correspondem, respectivamente, à densidade, dominância e frequência relativas.

Podemos observar que, Eschweilera coriaceae foi a de mais alto valor de importância, evidenciando ainda assim a importância desta espécie na área de pesquisa. Para Finol (1971) uma espécie é bem ajustada na estrutura e composto de uma floresta no momento em que se concentra em 
todos os estratos, sendo um bom indicador da conservação das espécies que se destacaram, caso os costumes de manejo tenham sido aplicados de maneira que não altere a dinâmica da mesma. $\mathrm{O}$ mesmo autor revela que a pesquisa da estrutura vertical é imprescindível no momento em que se trata de uma região em que se objetiva cumprir-se o manejo florestal.

A área apresentou a presença de espécies com alto valor comercial tais como Eschweilera coriacea, Tetragastris altíssima (Aubl.) Swart, Manilkara dardonai, Couratari oblongifolia Ducke \& R. Knuth, Goupia glabra e Dinizia excelsa, importantes para o manejo florestal madeireiro.
Condé e Tonini (2013) afirmaram que quando isso ocorre em uma área, geralmente a floresta se encontra em um estado avançado de sucessão ecológica.

Observa-se que a área de floresta estudada se caracterizou por uma grande quantidade de indivíduos concentrados em poucas famílias botânicas conforme pode-se observar pela figura 4, que apresenta graficamente as dez famílias com os maiores IVIF, com destaque para as famílias Fabaceae $(75,27 \%)$, Lecythidaceae $(59,88 \%)$ e Sapotaceae $(26,44 \%)$ que juntas perfazem cerca de $53,86 \%$ do índice de valor de importância familiar total.



Indice de valor de importancia familiar - IVIF

Figura 4. Parâmetros fitossociológicos das 10 famílias amostradas com maior valor de importância familiar. DR, DoR, e FR correspondem, respectivamente, à densidade, dominância e frequência relativas.

A família Fabaceae constitui uma das famílias botânicas de grande importância econômica e medicinal, destacando-se algumas espécies desta família no tratamento de doenças devido às suas propriedades curativas e terapêuticas. Muitas delas são usadas tanto na farmacologia quanto na medicina popular (Gomes et al. 2008).

Além da importância ecológica, a família também tem notável importância econômica na Amazônia. Atualmente, no Estado do Pará, 28 espécies de Lecythidaceae são exploradas para produção de madeira (Pará 2012).

Dentre os caracteres morfológicos diagnósticos da família Sapotaceae, destaca-se a presença de laticíferos, cujo látex de espécies da América do Sul (Manilkara sp.) foi extensivamente utilizado para produção de goma de mascar até ser substituído pelo análogo industrializado (Monteiro 2006).

A família Chrysobalanaceae possui diversas utilidades, como: fabricação de móveis e carpintaria; assoalhos e tacos; recuperação de áreas degradadas; alimentação humana; alimentação animal (Arvores do Sul do Amazonas 2017).

\section{Caracterização das parcelas amostrais}

A média do índice de H' foi de 3,96, com destaque para a parcela 4 que apresentou o maior valor com 4,07. Já a média para $\mathbf{J}$ foi de 0,84 e a parcelas que apresentaram melhores resultados foram as parcelas 3 e 4 , conforme pode-se observar pelos dados da tabela 4 .

Tabela 4. Número total de árvores, famílias e espécies de cada parcela amostral do inventário florestal, com o respectivo valor do índice de Shannon-Weaver (H') e Equabilidade de Pielou (J).

\begin{tabular}{cccccc}
\hline Parcela & Indivíduos & Famílias & Espécies & H' & J \\
\hline 1 & 396 & 29 & 96 & 3,91 & 0,86 \\
2 & 399 & 30 & 95 & 3,37 & 0,74 \\
3 & 419 & 33 & 103 & 4,01 & 0,87 \\
4 & 407 & 29 & 108 & 4,07 & 0,87 \\
\hline Média & 405,25 & 30,25 & 100,5 & 3,96 & 0,84 \\
\hline
\end{tabular}

O índice de Shannon, calculado para cada parcela, variou de 3,37 a 4,07. O índice da Equabilidade de Pielou (J) apresentou nas parcelas 1, 3 e 4 valores acima de 0,80 e na parcela 2 valor próximo a 0,74 , comprovando a existência de elevada diversidade florística na área estudada e baixa dominância das espécies.

A estrutura horizontal tem $53,79 \%$ dos indivíduos arbóreos até a classe diamétrica de 42,50 $\mathrm{cm}$ (figura 5), podemos observar ainda que existe uma grande concentração de árvores nas primeiras 


\section{Limeira et al.}

classes de diâmetro representando aproximadamente 91,54 \% dos indivíduos até o diâmetro máximo de $72,5 \mathrm{~cm}$. Conforme Machado et al. (2004), a grande quantidade de indivíduos nas classes de menor diâmetro pode indicar que o regime de perturbação é relativamente intenso e contínuo.

A floresta estudada não se mostra balanceada, ou seja, a constante "q" de Liocourt não permanece igual nas classes diamétricas, existindo uma grande quantidade de indivíduos menores e mais finos nas primeiras classes de diâmetro indicando que houve nessa área perturbação antrópica (Alves Junior et al. 2007). Nunes et al. (2003), ressaltam que a grande quantidade de indivíduos pequenos e finos, encontrados nas primeiras classes diamétricas, pode indicar a ocorrência de severas perturbações no passado. Carvalho e Nascimento (2009), afirmam que se $\mathrm{o}$ valor do quociente $\mathrm{q}$ for maior que $\mathrm{o}$ quociente q médio da população, há problemas com a estrutura diamétrica. Desta maneira, os quocientes q: q2 $(4,6), \mathrm{q} 3(1,7)$, q5 $(1,2)$ q6 $(2,4)$ q10 $(1,0)$ q12 $(1,7)$ q17(1,3) e q24 $(2,0)$, calculados apresentam valores superiores ao q médio calculado $(0,99)$, demostrando assim que nessas classes, a estrutura da floresta sofreu um desequilíbrio, por meio da existência de problemas no estabelecimento dos indivíduos, ou seja, essa alteração no número de indivíduos indica que nessas classes diamétricas ocorreram alta mortalidade. Para as demais classes os valores de $\mathrm{q}$ correspondentes às classes intermediárias e de maiores diâmetros, apresentam valores abaixo do q médio calculado $(0,99)$, constatando que nessas classes diamétricas não há problemas de regeneração, isto é, houve um equilíbrio entre o processo de recrutamento e mortalidade. A estrutura vertical (figura 5) mostra que $50,95 \%$ estão presentes nas quatro primeiras classes com altura de até $10,5 \mathrm{~m} ; 39,17 \%$ estão presentes nas classes de 11,5 a $15,5 \mathrm{~m}$ e $9,85 \%$ estão nas classes maiores que $15 \mathrm{~m}$.

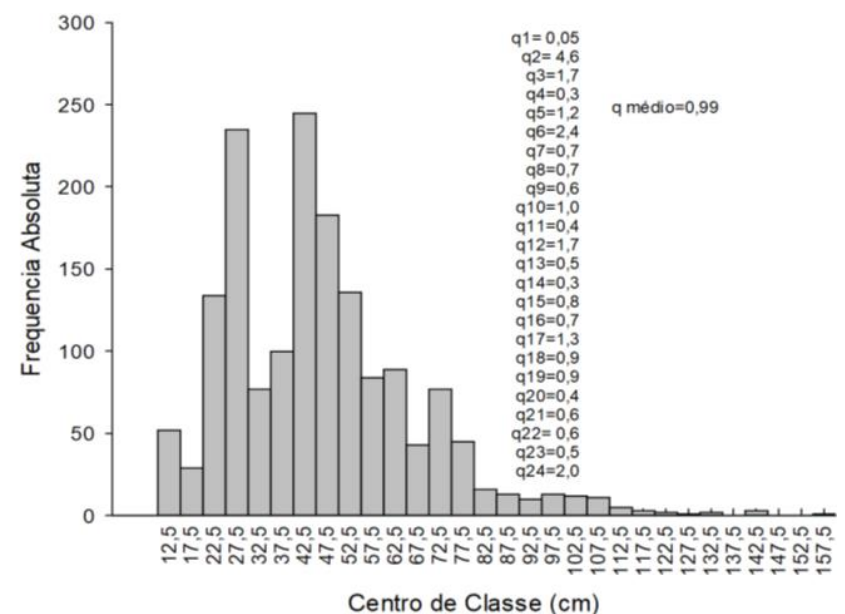

(a)

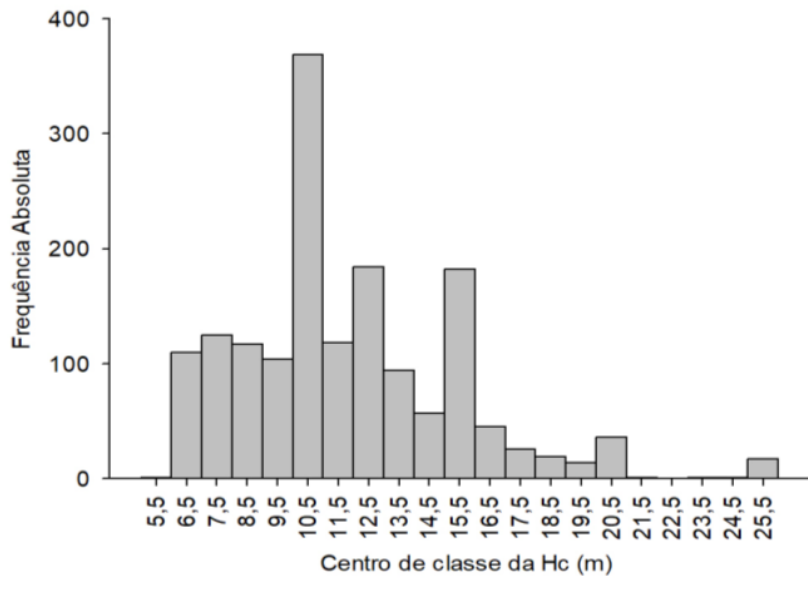

(b)

Figura 5. Distribuição diamétrica e valores do quociente "q" de Liocurt das classes diamétrica (a) e distribuição da altura comercial de todos os indivíduos amostrados na área de estudo (b)

O coeficiente de Sorensen (Sorensen 1948), é similar ao Jaccard, porem permitindo peso 2 para concordâncias. Caso as variáveis envolvidas sejam nulas e indefinidas, a aplicação do índice é não recomendada.

$$
S j=\frac{a}{2 a+b+c}
$$

Onde: $\mathrm{a}=$ número de espécies presentes nas duas áreas; $\mathrm{b}=$ número de espécies exclusivas da área/amostra $1 ; \mathrm{c}=$ número de espécies exclusivas da área/amostra 2.

O dendrograma para as parcelas (figura 6) onde o método utilizado foi o de média de grupo (UPGMA) tendo como base o índice de Sorensen, mostra que houve uma formação de dois grupos distintos entre as parcelas, onde o primeiro grupo está constituído somente pela parcela 2 e o segundo grupo pelas parcelas 1, 3 e 4. Este último grupo se subdividiu em dois outros grupos distintos, onde o primeiro é composto somente pela parcela 1 e o segundo composto pelas parcelas 3 e 4 . Indicando que existe similaridade entre as parcelas 1,3 e 4 , com nível de ligação em torno de $75 \%$ a $95 \%$ e há dissimilaridade da parcela $2 \mathrm{com}$ as demais parcelas, mas a similaridade analisada entre as áreas pode ser considerada como alta. Esse fator pode ser comprovado pelos resultados apresentados pelos índices de Shannon e pela equabilidade de Pielou, que apresentaram resultados próximos para as parcelas 1,3 e 4 deixando a parcela 2 como única.

Esse por sua vez, possui como importância a sua capacidade de demonstrar se as áreas estudadas possuem as mesmas características florísticas, como número de espécies em cada área. Segundo Ferreira Junior (2008) compreender a composição e similaridade florística de um ambiente permite o mapeamento da área e a obtenção de informações 


\section{Limeira et al.}

para elaboração de planos de manejo florestal para utilização sustentável do recurso.

\section{UPGMA}

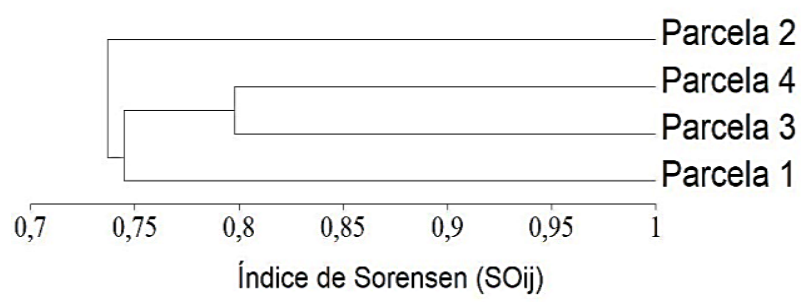

Figura 6. Dendrograma obtido a partir do Índice de Similaridade de Sorensen, para os dados de presença e ausência das espécies, nas 4 parcelas amostrais da área de estudo.
Observa-se ainda que as parcelas 3 e 4 se mostraram mais similares entre as demais. Nota-se ainda que estas parcelas foram as que tiveram os maiores valores do índice de H'. Ainda observando a mesma figura, vemos que o primeiro grupo composto unicamente pela parcela 2 mostrou-se a mais dissimilar das outras.

\section{Estrutura vertical}

Para a análise da estrutura vertical os dados da área foram divididos em três estratos de altura $(\mathrm{Ht}) . \mathrm{O} \mathrm{E} 1=$ estrato $1(\mathrm{Ht}<7,7 \mathrm{~m}), \mathrm{E} 2=$ estrato 2 $(7,7 \mathrm{~m} \leq \mathrm{Ht}<14,9 \mathrm{~m})$ e E3 = estrato $3(\mathrm{Ht} \geq 14,9 \mathrm{~m})$, conforme descritos na tabela 5 .

Tabela 5. Número de árvores por estrato de altura total $(\mathrm{H})$ conforme o índice de valor de importância, na área amostrada.

\begin{tabular}{l|ccccc}
\hline \multicolumn{1}{c}{ Espécie } & E1 & E2 & E3 & PSA & PSR \\
\hline Eschweilera coriácea & 19 & 164 & 15 & 111,4 & 14,33 \\
Licania macrophylla & 32 & 82 & 14 & 60,3 & 7,76 \\
Alexa grandiflora & 16 & 45 & 11 & 33,6 & 4,32 \\
Vouacapoua americana & 13 & 47 & 6 & 33,4 & 4,30 \\
Sagotia brachysepala & 10 & 40 & 5 & 28,2 & 3,63 \\
Macrosamanea pubiramea & 6 & 29 & 14 & 22,4 & 2,89 \\
Inga heterophylla & 0 & 31 & 5 & 21,0 & 2,70 \\
Goupia glabra & 1 & 22 & 26 & 19,7 & 2,54 \\
Anacardium giganteum & 1 & 27 & 5 & 18,5 & 2,39 \\
Dinizia excelsa & 9 & 25 & 0 & 17,4 & 2,24 \\
Macrolobium limbatum & 0 & 21 & 7 & 14,9 & 1,93 \\
Eschweilera amara & 4 & 15 & 12 & 12,7 & 1,64 \\
Eschweilera odora & 3 & 15 & 11 & 12,4 & 1,60 \\
Syzygiopsis oppositifolia & 9 & 16 & 1 & 11,8 & 1,52 \\
Neoxythece elegans & 1 & 11 & 11 & 9,5 & 1,23 \\
Manilkara dardanoi & 0 & 10 & 8 & 8,1 & 1,04 \\
Iryanthera grandis & 2 & 9 & 8 & 7,7 & 1,00 \\
Eschweilera alata & 0 & 9 & 9 & 7,6 & 0,99 \\
Couratari oblongifolia & 1 & 6 & 14 & 6,9 & 0,90 \\
Piptadenia gonoacantha (Mart.) J.f.macbr. & 2 & 7 & 8 & 6,4 & 0,83 \\
\hline
\end{tabular}

Em que: E1 = estrato inferior, E2 = estrato médio, E3 = estrato superior; PSA = posição sociológica absoluta e PSR = posição sociológica relativa; em ordem decrescente de PSR.

$\mathrm{Na}$ área estudada, 14,5\% estão concentrados no E1, $64,2 \%$ no E2 e $21 \%$ concentram-se no E3. A maioria dos indivíduos se encontraram no E2, seguidos pelo E3 e E1, isso indica que nesta área poucos indivíduos conseguem atingir o dossel e a maior riqueza se deu por espécies secundárias, seguido das espécies climax e pioneiras o que pode classificar esta floresta como madura e com uma boa heterogeneidade de espécies (Condé e Tonini 2013).

A espécie Eschweilera coriaceae teve a maior quantidade de n.ha- $^{1}$ no E2, sendo que nos estratos E1 e E2 a espécie com maior n.ha ${ }^{-1}$ foi Licania macrophylla. Podemos observar ainda que, Eschweilera coriaceae é a espécie que possui maior índice de posição sociológica, evidenciando ainda mais a importância desta espécie na área de estudo.

Para Finol (1971) uma espécie é bem estabelecida na estrutura e composição de uma floresta quando se concentra em todos os estratos, sendo um bom indicador da continuidade das espécies que se destacaram, caso as práticas de manejo tenham sido aplicadas de forma que não altere a dinâmica da mesma. $\mathrm{O}$ mesmo autor revela ainda que o estudo da estrutura vertical é fundamental quando se trata de uma área em que se objetiva realizar o manejo florestal. No presente trabalho, observou-se que apenas 6 espécies totalizaram $62,36 \%$ do valor total da posição 
sociológica, comprovando assim a importância dessas para a estrutura da floresta.

A área basal média por hectare é de 10,110 $\mathrm{m}^{2}$.ha ${ }^{-1}$ sendo que as oito espécies de maiores áreas basais se encontram nas espécies de maiores IVI, maiores abundâncias e maiores densidades sendo Eschweilera odora $\left(31,27 \mathrm{~m}^{2}\right)$, Licania macrophylla $\left(17,88 \mathrm{~m}^{2}\right)$, Buchenavia grandis, $\left(17,41 \mathrm{~m}^{2}\right)$, Goupia glabra $\left(11,56 \mathrm{~m}^{2}\right)$, Dinizia excelsa $\left(11,56 \mathrm{~m}^{2}\right)$, Vouacapoua americana $\left(9,95 \mathrm{~m}^{2}\right)$, Macrosamanea pubiramea $\left(9,45 \mathrm{~m}^{2}\right)$ e Anacardium giganteum $(9,21$ $\mathrm{m}^{2}$ ). Resultados muito semelhantes ao desse trabalho foram encontrados por Vieira et al. (2014) ao compararem a estrutura entre floresta manejada e não manejada em Santarém-PA encontraram na floresta manejada como espécie de maior importância a Eschweilera coriaceae, um índice de diversidade de Shannon de 4,42 e Equabilidade de Pielou (J) de 0,86 e uma área basal de $18,47 \mathrm{~m}^{2}$. ha 1. As famílias com maior riqueza de espécies em ordem decrescente, foram Fabaceae (41), Sapotaceae (13), Moraceae (11), Lecythidaceae (10) e Apocynaceae (8) que, juntas, contribuem com $48,2 \%$ da riqueza local, ficando as 35 famílias restantes, responsáveis por $51,7 \%$ do total, evidenciando a baixa abundância relativa de indivíduos nestas famílias.

Existem diversas famílias botânicas encontradas na Amazônia que apresentam elevado potencial econômico e dentre estas destaca-se as espécies de Fabaceae (477), Lecythidaceae (297), e Sapotaceae (159) e que, neste estudo estas mesmas famílias se destacam por apresentarem os maiores índices de valor de importância (IVI). Ao estudarem uma área de Floresta Ombrófila, no estado de Roraima, Condé e Tonini (2013) encontraram uma maior concentração de espécies nas famílias botânicas Fabaceae, Lecythidaceae e Sapotaceae, com 1.883, 609 e 434 indivíduos respectivamente, similar aos resultados encontrados nesta pesquisa.

No presente estudo, Fabaceae foi a família que apresentou a maior riqueza de espécies (41 espécies), dado este também apresentado em outros estudos realizados na Amazônia Legal por Gonçalves e Santos (2008), Carim et al. (2013), Almeida et al. (2012), Oliveira et al. (2008), Matos e Amaral (1999); Amaral et al. (2000), Lima Filho et al. (2001) e Oliveira e Amaral. (2004). Segundo Scudeller e Souza (2009) em um estudo feito na floresta de igapó da bacia do lago Tupé, na Amazônia, as famílias com maior número de espécies foram, Fabaceae (34 espécies), Myrtaceae (9 espécies) e Loranthaceae (7 espécies).

As espécies mais abundantes foram Eschweilera coriacea, Licania macrophylla, Vouacapoua americana, Sagotia brachysepala, Inga heterophylla, Alexa grandiflora, Macrosamanea pubiramea, Neoxythece elegans, Syzygiopsis oppositifolia, Eschweilera odora e Eschweilera amara que, juntas, somaram $44,42 \%$ dos indivíduos amostrados.

Vouacapoua americana e Syzyogiopsis oppositifolia apesar de terem obtido alta densidade, são consideradas em perigo de extinção, vulnerável a extinção e quase ameaçada, respectivamente (Martins e Martinelli 2013), fato que pode ser explicado por serem espécies de grande interesse comercial. Estas espécies também foram citadas por Das Flores et al. (2012) ao estudar uma unidade de produção anual (UPA) nesta mesma área, os autores encontraram estas mesmas espécies através da análise do inventário $100 \%$.

Vinte e cinco espécies (15, $06 \%$ da riqueza) apresentaram somente um indivíduo. Estas espécies mesmo não sendo exploradas, representaram $1,54 \%$ do total de indivíduos. A média geral para a variável DAP foi de $39,94 \mathrm{~cm} \pm 19,23 \mathrm{~cm}$ e coeficiente de variação $=48,14 \%$ e para variável altura comercial a média foi de $10,96 \mathrm{~m} \pm 2,94 \mathrm{~m}$ e coeficiente de variação $=26,8 \%$ sendo estes considerados valores altos, classificando as áreas como heterogêneas.

Dentre as espécies comerciais mais colhidas para produção de madeira no Pará, encontra-se a Eschweilera coriacea, esta espécie caracterizada como clímax tem alto valor comercial e é encontrada na área a uma densidade de 6,19 ind.ha- ${ }^{1}$. Valor muito inferior ao encontrado por Alvino et al. (2005) de 77 ind.ha- ${ }^{1}$ em Bragantina-PA em área de floresta secundária e 30 anos de sucessão, pertencente a um agricultor familiar e proveniente da área abandonada após sucessivos cultivos anuais de milho, mandioca e arroz.

Para Licania macrophylla, espécie considerada clímax, a densidade é de 4 ind.ha- $^{1}$ e a estrutura horizontal mostrou que $27,34 \%$ dos indivíduos estão no intervalo de $12,5-32,5 \mathrm{~cm}, 55,47$ $\%$ entre $32,5-52,5 \mathrm{~cm} 17,19 \%$ maior que $50 \mathrm{~cm}$. Para a estrutura vertical $62,5 \%$ então entre $6,5-10,5 \mathrm{~m}$; $26,56 \%$ entre $11-14 \mathrm{~m} ; 8,6 \%$ maior que $14 \mathrm{~m}$. A média do DAP é de $39,86 \mathrm{~cm}$ e desvio padrão de $13,74 \mathrm{~cm}$. Para altura total a média é de $10,09 \mathrm{~m}$ com desvio de 3,02m.

Em sua estrutura horizontal a espécie Vouacapoua americana, com a densidade 2,25 ind.ha- ${ }^{1}$ apresentou que $31,90 \%$ dos indivíduos estão entre 10 e $30 \mathrm{~cm}, 52,73 \%$ entre 30 e $50 \mathrm{~cm}$ e $15,32 \%$ estão maiores que $50 \mathrm{~cm}$ de DAP, sendo a média de DAP de 39,00 cm com um desvio padrão de 15,42 $\mathrm{cm}$. Já em sua estrutura vertical essa espécie teve $68,05 \%$ dos indivíduos entre 6 e $10 \mathrm{~m} ; 16,67$ entre 11,5 e $14,5 \mathrm{~m}$ e $15,28 \%$ entre 14,5 e $16,5 \mathrm{~m}$ de altura, sendo a média de alturas foi de $9,93 \mathrm{~m}$ com um desvio padrão de $3,01 \mathrm{~m}$.

Para a espécie Sagotia brachysepala, com densidade de 2,06 ind.ha- ${ }^{1}$, sua estrutura horizontal teve $51,51 \%$ dos indivíduos entre 12,5 e $32,5 \mathrm{~cm}$; $33,33 \%$ ente 32,5 e $55,5 \mathrm{~cm}$ e $15,15 \%$ dos indivíduos com DAP maiores que $35,5 \mathrm{~cm}$, sendo a média e o 


\section{Limeira et al.}

desvio padrão de $59,20 \mathrm{~cm}$ e $12,92 \mathrm{~cm}$, respectivamente. Já em sua estrutura vertical essa espécie teve 53,03\% dos indivíduos entre 6,5 e 10,5 $\mathrm{m} ; 37,9 \%$ entre 1,51 e $14,5 \mathrm{~m}$ e $9,07 \%$ entre 14,5 e $16,5 \mathrm{~m}$ de altura, sendo a média e o desvio padrão de $10,35 \mathrm{~m}$ e $2,61 \mathrm{~m}$, respectivamente.

A estrutura horizontal de Inga heterophylla com a densidade de 1,72 ind.ha- ${ }^{1}$, mostrou que $56,36 \%$ dos indivíduos estão entre 12,5 e $32,5 \mathrm{~cm}$; $21,81 \%$ entre 32,5 e 52,5 e $21,82 \%$ com DAP maiores que $52 \mathrm{~cm}$. Já a estrutura vertical da mesma teve $67,27 \%$ dos indivíduos entre 6,5 e $10,5 \mathrm{~m} ; 20 \%$ entre 11,5 e $13,5 \mathrm{~m}$ e $11,73 \%$ entre 14,5 e $16,5 \mathrm{~m}$ de altura. As médias de DAP e altura foram de 35,18 $\mathrm{cm}$ e 10,21 m, respectivamente. Já os valores de desvio padrão foram de $16,34 \mathrm{~cm}$ e 3,11 , sendo DAP e altura.

Os índices de diversidade e de equabilidade mostraram que a área estudada apresenta diversidade e riqueza altas mostrando desta forma que $o$ gradiente de riqueza e diversidade tende a ficar equilibrado caso não ocorram perturbações ao longo do tempo. Ao analisarmos a tabela 2 observamos que a média do índice de diversidade entre as parcelas foi de 3,96, resultado melhor que o encontrado por Silva et al. (2008) que encontraram um valor de 2,71 em Manaus-AM e por Condé e Tonini (2013) que encontraram um valor de 3,27 no estado de Roraima. Comparado a outros estudos na Amazônia Legal, como o de Almeida et al. (2012), que ao estudar uma área de Floresta Ombrófila Densa manejada em Santarém-PA, obteve um índice de H' de 4,39, maior que o encontrado neste estudo. Esta diferença entre florestas tropicais pode ocorrer devido existir uma maior dominância de algumas espécies dentro da comunidade e por uma menor homogeneidade florística quanto ao nível de gênero (Malheiros et al. 2009) enquanto que para equabilidade de Pielou (J) os resultados foram semelhantes ao encontrado por Almeida et al. (2012) em Santarém, Pará.

\section{Conclusões}

A distribuição diamétrica para a comunidade arbórea e para as espécies de maior IVI apresentaram padrão típico de distribuição para florestas normais.

A área estudada apresenta uma alta diversidade florística no estrato arbóreo, sendo concentrada nas famílias Fabaceae, Lecythidaceae e Sapotaceae.

Os índices de equidade mostraram igualdade na composição das parcelas e foram bastante representativos destas. A similaridade analisada entre as áreas pode ser considerada como alta. A área apresenta distribuição desbalanceada ao analisar os valores do quociente de Liocourt. Houve tendência de taxas de recrutamento e mortalidade bastante variáveis.

A extração de madeira provocou alterações na abertura do dossel, mas não foram constatadas diferenças significativas no número de espécies e famílias.

As espécies são parte de mesma tipologia florestal, e o manejo, mesmo de baixo impacto, afetou o padrão de distribuição espacial de algumas espécies arbóreas. Para espécies com padrão agregado, devem-se manter alguns indivíduos de tamanho comercial, para que continue a ocorrer a distribuição natural. A área apresenta potencial para a exploração madeireira, desde que haja manutenção da diversidade, densidade e área basal de forma a garantir a sustentabilidade da floresta.

\section{Referências}

Almeida LSD, Gama JRV, Oliveira FDA, Carvalho JOPD, Gonçalves DCM, Araújo GC (2012) Fitossociologia e uso múltiplo de espécies arbóreasagregag em floresta manejada, Comunidade Santo Antônio, município de Santarém, Estado do Pará. Acta Amazonica, 42(2): 185-194.

Alvares CA, Stape JL, Sentelhas PC, De Moraes G, Leonardo J, Sparovek G (2013) Köppen's climate classification map for Brazil. Meteorologische Zeitschrift, 22(6):711-728.

Alves Júnior FT, Lins CF, Brandão S, Rocha KD, Silva JT, Maragon LC, Caraciollo RLF (2007) Estrutura diamétrica e hipsométrica do componente arbóreo de um fragmento de mata atlântica, RecifePE. Cerne 13(1): 83-95.

Alvino FO, Silva MFF, Rayol BP (2005) Potencial de uso das espécies arbóreas de uma floresta secundária, na Zona Bragantina, Pará, Brasil. Acta Amazonica, Manaus, 35(4): 413-420.

Amaral IL, Matos FDA, Lima J (2000) Composição florística e parâmetros estruturais de um hectare de floresta densa de terra firme no Rio Uatumã, Amazônia, Brasil. Acta amazonica, 30(3): 377-392.

APG III (2009) An update of the Angiosperm Phylogeny Group classification for the orders and families of flowering plants: APG III. Botanical Journal of the Linnean Society, 161: 105-121.

Árvores Do Sul Do Amazonas (2017) - Guia De Espécies De Interesse Econômico E Ecológico, 2: 57.

Batista APB, Aparicío WCS, Aparicio PS, Santos VS, Lima RB, Mello JM (2015) Caracterização estrutural em uma florestal de terra firme no estado do Amapá, Brasil. Pesquisa Florestal Brasileira, 35(81): 21-33.

Carim MJV, Guillaumet JLB, Guimarães JRS, Tostes LCL (2013) Composição e estrutura de 
floresta ombrófila densa do extremo norte do estado do Amapá, Brasil. Biota Amazônia (Biote Amazonie, Biota Amazonia, Amazonian Biota), 3(2): 1-10.

Carvalho FA, Nascimento MT 2009 Estrutura diamétrica da comunidade e das principais populações arbóreas de um remanescente de floresta atlântica submontana (Silva Jardim-Rj, Brasil). Revista Árvore, Viçosa-MG, 33(2): 327-337.

Castro MAS, Viana APS, Lobato CC, Muniz JL, Lima MWS, Melo LO (2018) Impactos da exploração florestal na estrutura e na composição florística da vegetação remanescente em área manejada na floresta nacional do Tapajós, Belterra, Pará. Revista Agroecossistemas, 10(2): 125-135.

Condé TM, Tonini H (2013) Fitossociologia de uma floresta ombrófila densa na Amazônia Setentrional, Roraima, Brasil. Acta Amazonica. 43(3): 247-260.

Das Flores OMM, de Queiroz WT, Pinheiro JG, Oliveira FA, Moraes A, Valente MDR (2012) Comparação de tamanhos e distâncias de subparcelas aplicadas em processo de amostragem por conglomerado. Acta Amazonica, 42(3): 345354.

De Queiroz WT, Netto SP, Valente MDR, Pinheiro JG (2011). Análise estrutural da unidade conglomerada cruz de malta na Floresta Nacional do Tapajós, estado do Pará, Brasil. Floresta, 41(1).

Dos Santos ES, Aparicio PS, Silva TL, Freitas JL (2013) Distribuição diamétrica para Virola surinamensis (Rol.) Warb na Floresta Estadual do Amapá-Flota/AP. Revista de biologia e ciências da terra. Aracaju, 13(1): 34-47.

Ferreira Junior EV, Soares TS, Costa MFF da, Silva VSM (2008) Composição, diversidade e similaridade florística de uma floresta tropical semidecídua submontana em Marcelândia MT. Acta Amazônica, 38(4): 673-679.

Finol UH (1971). Nuevos parametros a considerarse en el análisis estrutural de las selvas vírgenes tropicales. Revista Forestal Venezolana, 18: 12. 2942.

Fisch G, Marengo JA, Nobre CA (1998) Uma revisão geral sobre o clima da Amazônia. Acta amazônica, 28(2): 101-126.

Gomes ECS, Barbosa J, Vilar FCR, Perez JO, Vilar RC, Freire JLO, Lima NA, Dias TJ (2008). Plantas da Caatinga de uso terapêutico: Levantamento
Etnobotânico. Engenharia Florestal, Espirito Santos do Pinhal 5: 74-85.

Gomes FP (1990). Curso de estatística experimental. 12.ed. São Paulo: Nobel, 467p.

Gonçalves FG, Santos J (2008) Composição florística e estrutura de uma unidade de manejo florestal sustentável na Floresta Nacional do Tapajós, Pará. Acta Amazonica, 38(2): 229-244.

IBGE (2012) Manual técnico da vegetação brasileira. Rio de Janeiro: IBGE.

Junk WJ, Piedade MTF, Schöngart J, Cohn-Haft M, Adeney JM, Wittmann F, (2011). A classification of major naturally-occurring Amazonian lowland wetlands. Wetlands, 31(4): 623-640.

LAMPRECHT H (1962) Ensayo sobre unos metodos para el análisis estructural de los bosques tropicales. Acta Cientifica Venezoelana, 13(2): 57 65.

Lima Filho DA, Matos FDA, Amaral IL, Revilla J, Coêlho LS, Ramos JF, Santos JL (2001) Inventário florístico de floresta ombrófila densa de terra firme, na região do Rio Urucu-Amazonas, Brasil. Acta Amazonica, 31(4): 565-579.

Machado ELM, Filho ATO, Carvalho WAC, Souza JS, Borém RAT, Botezelli, L (2004) Análise Comparativa da Estrutura e Flora do Compartimento Arbóreo-Arbustivo de um Remanescente Florestal na Fazenda Beira Lago, Lavras, MG. Revista Árvore, Viçosa-MG, 28(4): 499-516.

Malheiros AF, Higuchi N, Santos J (2009) Análise estrutural da floresta tropical úmida do município de Alta Floresta, Mato Grosso, Brasil. Acta Amazonica, 39(3): 539-548.

Martins EM, Martinelli G (2013) Listas Vermelhas e Estratégias de Conservação: a atuação do Centro Nacional de Conservação da Flora (CNCFlora) com as espécies da Flora ameaçadas de extinção. II Simpósio sobre a biodiversidade da mata atlântica. Rio de Janeiro, 23-28.

Matos FDA, Amaral ID (1999). Análise ecológica de um hectare em floresta ombrófila densa de terrafirme, estrada da várzea, Amazonas, Brasil. Acta Amazonica, 29(3), 365-379.

Monteiro MHDA (2006). Taxonomia e anatomia das espécies de Pouteria Aublet (Sapotaceae) do estado do Rio de Janeiro, Brasil. Tese de doutorado, Curso de pós-graduação em Ciências biológicas (botânica). Rio de Janeiro: UFRJ. 123p. 
Mueller-Dombois D, Ellenberg H (1974) Aims and methods of vegetation ecology. 573p.

Nunes YRF, Mendonça AVR, Botezelli L, Machado ELM, Filho ATO (2003) Variações da Fisionomia, Diversidade e Composição de Guildas da Comunidade Arbórea em um Fragmento de Floresta Semidecidual em Lavras, MG. Acta Botanica Brasilica, 17(2): 213-229.

Oliveira AD, Amaral ID, Ramos MBP, Nobre AD, Couto LB, Sahdo RM (2008) Composição e diversidade florístico-estrutural de um hectare de floresta densa de terra firme na Amazônia Central, Amazonas, Brasil. Acta Amazonica, 38(4): 627-642.

Oliveira AN, Amaral IL (2004) Florística e fitossociologia de uma floresta de vertente na Amazônia central, Amazonas, Brasil. Acta Amazonica, 34(1): 9-35.

PARÁ. Secretaria de Estado de Meio Ambiente. Extração e Movimentação de Toras de Madeira Nativa: 2006 a 2012. Disponível em: <http://monitoramento.sema.pa.gov.br/sisflora/>.

Acesso em: 15 de out. 2020.

Pereira LA, Pinto Sobrinho FA, Costa Neto SV (2011) Florística e estrutura de uma mata de terra firme na Reserva de Desenvolvimento Sustentável Rio Iratapuru, Amapá, Amazônia Oriental, Brasil. Floresta, 41(1): 113-122.

Perigolo NA (2014) Caracterização dos tipos vegetacionais do médio rio madeira, Rondônia. Dissertação de mestrado, Departamento de Botânica do Instituto de Ciências Biológicas, Universidade de Brasília, 78p.

Pielou EC (1966) Species-diversity and patterndiversity in the study of ecological succession. Journal of theoretical biology, 10(2): 370-383.

Scudeller VV, Souza AM (2009) Florística da mata de igapó na Amazônia Central. Ed: UEA. BioTupé: Meio Físico, Diversidade Biológica e Sociocultural do Baixo Rio Negro, Amazônia Central, Manaus, p. 97-108.

Silva KE, Matos FDA, Ferreira MM (2008) Composição florística e fitossociologia de espécies arbóreas do Parque Fenológico da Embrapa Amazônia Ocidental, Acta Amazonica, 38: 213-222.

Soares CPB, Neto FP, Souza AL (2006) Dendrometria e Inventário Florestal. Viçosa: Ed. UFV, 276p.
Souza DR, Souza AL, Gama JRV, Leite HG (2003). Emprego de análise multivariada para estratificação vertical de florestas inequiiâneas. Revista Árvore, 27: 59-63.

Sorensen T (1948) A method of establishing groups of equal amplitude in plant sociology based on similarity of species content and its application to analyses of the vegetation on danish commons, Vidensk Selsk Biol Skr, 5, 1-34.

Vieira DS, Gama JRV, Ribeiro RBS, Ximenes LC, Correa VV, Alves AF (2014) Comparação estrutural entre floresta manejada e não manejada na comunidade Santo Antônio, Estado do Pará. Ciência Florestal [online], 24(4): 1067-1074. doi: org/10.1590/1980-509820142404025.

Wittmann F, Householder E, Piedade MTF, Assis RL, Schöngart J, Parolin P, Junk WJ (2013) Habitat specifity, endemism and the neotropical distribution of Amazon white-water floodplain trees Ecography (Cop.), 36: 690-700. 